\title{
Cancer care delivery in India at the grassroot level: Improve outcomes
}

Having spent more than a decade treating cancer patients abroad and a decade in India, I see no insuperable barrier to deliver the same quality of care to cancer patients in India as in the West provided there is coordination at all levels. As corporate/private cancer treatment centers, which are huge revenue generators, spring up all over the country, the lack of core guidelines to define a cancer center is a matter of concern. There is also a fear that the desire of ordinary Indians to do the best for loved ones with a perceived "fatal" illness is being exploited as a recent report has highlighted corruption within the healthcare system. ${ }^{[1]}$ This is not to say, however, that a great deal of good does not exist and that a number of institutions both in the corporate and public sectors are delivering excellent care.

Cancer in India has to be a priority for the government at all levels. There are one million new cancer cases diagnosed every year in India, and this is expected to rise 5-fold by $2025 .^{[2]}$ Early detected cancers are curable and less expensive to treat and the patient goes back into the society to be productive. Lancet Oncology commissioned a group of Indian oncologists and eminent authors from across the globe to look at the drivers of cancer control in India. ${ }^{[3-5]}$ The major hindrances to early diagnosis and treatment were attributed to misinformation, absence of knowledge, low trust in public health services, and inadequate infrastructure, with $80 \%$ of health expenditure occurring in the private sector. ${ }^{[3]}$

If a coordinated effort is to be undertaken all over the country, a three-pronged approach needs to be made:

1. Prevention/education,

2. Service delivery, and

3. Research, all in parallel.

The Ministry of Health and Family Welfare with both its secretariat and technical divisions that include the department of health, the department of family welfare and the directorate of health services, need to work

\begin{tabular}{|l|l|}
\hline \multicolumn{2}{|c|}{ Access this article online } \\
\hline Quick Response Code: & Website: \\
\hline & Www.jmpo.org \\
\cline { 2 - 2 } & \\
\hline
\end{tabular}

together. They need to identify a cancer nodal person who coordinates between all these departments toward a common goal of increasing 5-year survival from cancer which currently stands at 30\% in India, compared to $60 \%$ in the West. ${ }^{[6]}$ For a center to be labeled as a "cancer center," they should be able to or have the desire to be able to deliver on all of the following within 12 months of starting as a center.

\section{PREVENTION/EDUCATION}

The old English proverb "prevention is better than cure" applies to cancer. Certain cancers are amenable to primary and secondary prevention. This would mean increasing awareness and education programs targeting promotion of healthy lifestyles, reduced tobacco use, investment to change social attitudes and personal habits, improvement of cancer registries, and mass screening for some cancers. The delivery of this has to be prioritized.

Some of the following may be made mandatory for the centers to be classified as cancer centers with different grading based upon their performance - level 1,2, and 3 cancer centers with level 3 being ideal (optimal).

\section{Adopting villages}

According to the last Census in 2011, $72 \%$ of India's population live in villages. Thus, the main focus has to be directed at the villages in tandem with the urban areas. ${ }^{[7]}$ Cancer centers have to take the lead in committing to adopt at least 10-15 villages in their vicinities for cancer education, awareness, and clinical check-ups. I have gone into villages in UP, Rajasthan, and Punjab, and this exercise costs nothing apart from travel costs. Cancer specialists/ centers have an obligation to educate the public, the primary care physicians, and other specialties (ENT, dental, gynecology forum, chest physicians, nephrologists, etc.) so that appropriate early referrals and timely treatments are given to patients.

\section{Unified guidelines and treatment protocols}

Various professional bodies and some cancer centers have come up with guidelines for cancers, but it is important that all centers follow one set of guidelines. The Indian Council of Medical Research has done a wonderful job of coming up with site specific consensus statements for various cancers, and these can be easily implemented 
country wide. The diagnostic tests, treatment etc., have been categorized as essential or desirable/ideal-essential is the bare minimum requirement if a patient is being treated for cancer and should be offered to all patients undergoing treatment at the center; desirable/ideal are tests and treatments that may not be available at all centers but the centers should aspire to have them in near future. ${ }^{[8]}$ More importantly, if the ideal/desirable is the treatment of choice and is unavailable at a particular site, the treating oncologists should be aware of centers with the ideal and are obliged to refer the patient there.

Preventive screening and diagnosis of common cancers should be part of the teaching curricula at the undergraduate level. Implementation of these guidelines will be a challenge but is doable if it is an incentive driven. An effort from the National cancer grid described in this issue by Pramesh et al. to do the same with simplified algorithms for diagnosis and treatment will make this easier to implement..$^{[9]}$

\section{Communication skills}

For some cancers such as lung, gall bladder, and pancreatic cancer, a performance status of 2 or more indicates a survival of $<6$ months. Do patients want to spend this time undergoing chemotherapy and sometimes weekly blood tests? The balance between informed patient choice, qualityof-life, and risk-benefit ratio needs to be reached between the patient and the oncologist. Training at undergraduate, postgraduate, or even specialist level must stress on how to break bad news, how to discuss no treatment as a reasonable option with the patient, communicating with children, supporting psychosexual issues, the role of hope, etc. This needs communication skill training. The helps refine the "art of caring" for a cancer patient.

\section{Patient power}

Patient choice and control have to be at the core of cancer treatment pathway. The Royal College of Physicians has set up a patient-caregiver network to improve the healthcare delivery by supporting shared decision-making which takes into account not only the professional evidence but the patient's own preferences. ${ }^{[10]}$ It is important to have patient participation in the decision-making.

Preventing common cancers (head neck, cervix, breast, and lung cancer)

1. Smoking/tobacco cessation clinics should be encouraged at all cancer centers as this will help prevent $40 \%$ of tobacco-related cancers. ${ }^{[1]}$ All health care workers - nurses, pharmacists, paramedics, and undergraduate students must have tobacco and health as a mandatory course curriculum.

2. Sexual hygiene is one of the key risk factors for cervical cancer. The initiative taken by some state governments and the current central government, as part of the Clean India Campaign/Nirmal Bharat Abhiyan (Tamil $\mathrm{Nadu}$ ), to provide toilets in all schools and villages will help toward this significantly. Another strategy that is easily implementable with minimal training to multipurpose health workers is the VIA/vinegar test for rural population to screen for cervical cancer as this has been shown to save lives. ${ }^{[12,13]}$

3. National cancer registry program is doing a commendable job with the collection and publication of cancer registry data. This will help stakeholders to optimize screening strategies for population at highrisk for certain types of cancer in different parts of the country. ${ }^{[14]}$ For example, epidemiologic studies for certain cancers in areas of high incidence-colorectal cancer in Goa, stomach cancer in southern and northeastern states, gall bladder cancer in the Ganges belt, etc., may yield clues to prevention.

4. Education about dietary patterns, active lifestyle with physical activity, and breast self-examination (being breast aware), should be taught in schools, in villages, (through the gram panchayat, Anganwadi, and accredited social health activist workers) and in semi-urban/urban areas (through the resident welfare associations and other societies) at various levels.

5. Clinical screening for oral cancer especially in high-risk population (chewing tobacco) — visual examination, toluidine blue staining. ${ }^{[15]}$

\section{SERVICE-DELIVERY OF HIGH QUALITY CARE}

\section{Transparency/accountability}

Dreze and Sen, leading economists, elaborated in their book $^{[16]}$ on why health and education remain at the bottom of national priorities for India. According to them, "lack of transparency" and "lack of accountability" in the delivery of care at all levels are the main causes. The concept of reporting and discussing critical, never, and sentinel events in a transparent fashion within a hospital and learning from the mistakes made is by and large nonexistent (the audit process). While noted on paper, in most institutions, if these are not reported or are underreported, no lessons can be learnt. This is something that needs urgent attention. Increased rates of reporting errors or near misses within the healthcare setup lead to better patient care and safety; at present this is inhibited by the fear of litigation (risk management). Until reporting, these events on a regular (monthly or quarterly) basis is not made mandatory for hospitals in India, we will not improve the delivery of quality care and safety, as we will not have our own benchmark. A study has shown that nondisclosed errors lead to reduced trust and patient satisfaction. ${ }^{[17]}$ 


\section{Governance}

Clinical governance both in the private and the public sector is the desperate need of the hour. This has five major components - education, risk management, clinical audit, evidence-based care and effectiveness, patient and caregiver involvement. This makes sure that there is a systematic approach to maintain and improve the quality of patient care as "accountability of the organization" is at the core of clinical governance. ${ }^{[18]}$

\section{Multidisciplinary care}

The main barriers to a multidisciplinary team (MDT) care in India are logistics, education, cultural issues, and financial. Most secondary care centers do not have the MDT quorum due to lack of trained personnel and lack of facilities like video-link to tertiary centers. The cultural history and microenvironment does not lend itself well to collaborative approach between various disciplines. The solution to this is to educate the professionals and the policy makers.

All newly diagnosed cancer patients must be discussed by an MDT or the tumor board. These meetings should include a medical oncologist, radiation oncologist, surgical oncologist, radiologist, pathologist, nurse, and palliative care physician (optional though desirable). The patient and family may also be involved when necessary. If such a quorum is not available at every center, these discussions could be done by video link with centers that do have access to all the disciplines of oncology. This should be mandatory for all cancer centers as it will ensure that patient care is evidence-based and unbiased. ${ }^{[19]}$ These meetings will also help identify patients suitable for clinical research.

\section{Service reconfiguration}

Currently, in India, doctors from any discipline including surgery are able to give chemotherapy and cancer-targeted therapy without any legal hurdles. Surgical oncologists, radiation oncologist, and urologists routinely give hormone therapy and chemotherapy. This should only be done by trained medical oncologists in the best interest of the patients and the case for this is being made by the Indian Society of Medical and Pediatric Oncology. Till there are enough trained medical oncologists across the country, patients should be treated at specialist regional cancer centers.

Data show that high volume centers give best results for outcomes, with lower morbidity and mortality for all aspects of patients care especially complex surgeries, radiation therapy planning and highly complex chemotherapy protocols; this was the basis for the reconfiguration of services in the UK. ${ }^{[20]}$ Site-specific cancer surgery can only be done in high-volume centers and may be impractical for India to implement at all levels but for complex surgeries, the patients will get optimal care only if they are referred early to a specialist center. ${ }^{[21]}$

\section{Oncology clinical pharmacists}

Chemotherapy is a very high-risk medication that needs to be handled with care. This will take time, but we have to encourage the concept of specialist pharmacists for admixing chemotherapy under laminar flow hoods specific to drug reconstitution. ${ }^{[2]}$ This is not only for the safety of the person handling the medication but also reduces the microbiological contamination. In most hospitals, this is currently done by nurses who need to focus on administration and clinical aspects.

\section{Oncology nursing}

Trained clinical nurse specialists (CNS) provide high-quality and cost-effective care to cancer patients. ${ }^{[23]}$ The original concept of nurse consultants in the UK has been successful in developing and maintaining quality practice and services. The role of the CNS should be integrated into the cancer centers manpower requirements to begin with and over a period of time, at least for high volume centers, this could evolve into site specific CNSs. This needs training and education of nurses who are currently already delivering the care for cancer patients.

\section{End-of-life care}

This needs to be optimized for cancer patients. "Do not resuscitate" orders in the notes of patients with advanced cancer who are terminally ill is legal in most western countries but is not yet legal in India. This needs a legislative effort. Resuscitation in these situations does not help patients; in most cases it prolongs their agony. It is possibly due to the heroic culture of "fighting till the end" and lack of awareness among us physicians. Given that most Indian patients spend out of their pocket, it is very important to discuss end-of-life care with patients and families so the limited resources can be used to optimum. As physicians, we have a moral and legal obligation to inform the patient about the futility of further treatment if that is the case. We also have data from the west that show that hospice and palliative care is less expensive than hospital care. ${ }^{[24]}$

\section{Cost-effective care}

The cost of cancer management is mainly on the initial diagnostic tests, cancer drugs and end-of-life care. ${ }^{[25]}$ These costs are higher in India as majority of patients are diagnosed at advanced stages due to lack of adequate awareness and no coordinated screening strategy driven by the government. If the standard guidelines are followed, this would help in providing cost-effective care. A body like the UK National Institute of Clinical Excellence is needed in India which not only looks at the intervention or guidance but also if it is cost-effective. One can argue that this would 
not be in the best interest of the country as healthcare is not universally provided for Indian citizens. This can at least help us to optimize the use of certain drugs/interventions for some indications for e.g., patients with human epidermal growth factor- 2 positive breast cancer can at least get 9 weeks of therapy as shown in the FINHER trial rather than 12 months as is the standard all across the developed world. ${ }^{[26]}$ Controlling cost of drugs, compulsory licensing, royalty payment to innovator, and wiser use of the generic drugs will all help lower treatment costs.

Emphasis must be made to encourage governmental support to indigenization of diagnostic tests, expensive equipment (diagnostic and therapeutic) and perhaps through a public-private partnership.

\section{Databases}

In considering progress in dealing with cancers, it is usual for us to consider incidence, prevalence, mortality, and survival. It is data pertaining to the latter two that is sorely lacking from Indian cancer centers. It is important for each center to prospectively maintain databases for all patients so that key outcome measures are available to the policy makers.

\section{Key outcome measures}

As part of the service commitment, the following should be collated and published centrally on a periodic basis:

1. Stage at diagnosis.

2. Treatment offered: Chemotherapy, surgery, radiotherapy, palliation, and symptom control.

3. 30-day mortality post-surgery or radiation or chemotherapy.

4. Post-surgery morbidity for each cancer surgery.

5. Response rate to chemotherapy.

6. Acute and chronic toxicity post radiotherapy and chemotherapy.

7. Hospital acquired infection rate.

8. Cancer survival (1-year, 2-year...).

9. Adherence to guidelines.

\section{Choosing wisely}

A stop needs to be made to some practices currently being followed. Some of these are chosen from the ASCO Choosing Wisely articles: ${ }^{[27,28]}$

a. Screening all females in villages through mammography vans: Young women have dense breasts, and mammography will not help detect cancers early. Mammography has to be used wisely or not at all as it can give unnecessary radiation. Data clearly indicates that it should be done after menopause when breasts are fattier.

b. Shifting patients to intensive care units at the end-oflife when all cancer treatments have been exhausted.
These patients should be treated at home or local hospices.

c. Use of Image-guided and intensity modulated radiotherapy which are quite expensive for palliation.

d. Giving expensive targeted therapy (e.g. bevacizumab, cetuximab, panitumumab) to patients with advanced cancers for a few cycles, usually till their resources are exhausted. If patients cannot afford these therapies, which are of limited value, they should not be commenced. Targeted therapy should be used only where a specific biomarker is identified.

e. Genetic testing without adequate counseling: As a result, blood tests are being done to detect cancer.

f. Unnecessary tests like positron emission tomography (PET) scans/tumor markers as part of cancer screening packages, using PET scans in the staging of early prostate cancer at low risk of metastases (T1c) T2a prostate specific antigen $[\mathrm{PSA}]<10$ ) or for staging of early breast cancer at low risk of metastases or as part of routine cancer follow-up in asymptomatic patients. Do not do PSA levels in patients with no symptoms and a life expectancy of $<10$ years.

g. Using the cancer directed therapy in patients in whom there will be no benefit for e.g., those with performance status of 3-4, no benefit from prior evidence-based interventions, for example, use of the third line and beyond treatments for patients with advanced gallbladder and stomach cancers.

h. Using granulocyte colony-stimulating factor for primary prophylaxis of febrile neutropenia in those with $<20 \%$ risk of complication.

i. Giving high-risk anti-emetic regimens to those with low or moderate risk of developing nausea and vomiting with chemotherapy.

j. Using combination chemotherapy for the treatment of patients with metastatic breast cancer unless rapid response is needed for symptom control.

k. Offering cervical cancer vaccine to women who are unlikely to benefit from the same.

1. Cervical smears in women who are over 65 years of age.

\section{RESEARCH}

Research can only be built on the provision of sound, safe, evidence-based "service." I have already elucidated on research in India, and this needs a lot of work. ${ }^{[29]}$

How can we improve if we do not have our own baseline outcomes documented for treatment related morbidity, mortality, response rates, toxicity, and survival? We have to focus on reducing cancer incidence and on improving survival of our patients which will ultimately reduce 
cancer-related mortality. The creation of the National Cancer Grid of India in 2012 (a partnership of all the major regional cancer centers across India) and the drive to improve the quality of services across the public sector provides a major opportunity to improve cancer outcomes but it needs to be practically implemented as laid out above.

We as oncologists need to be more accountable and take greater responsibility for the entire cancer journey of each patient. It is the delivery of quality evidencebased care to each and every cancer patient, with unified protocols across the country, within limited resources, that is the key.

Bhawna Sirohi

Department of Medical Oncology, Kiran Mazumdar-Shaw Cancer Centre, Narayana Health, Bengaluru, Karnataka, India. E-mail: bhawna.sirohi13@gmail.com

\section{REFERENCES}

1. Berger D. Corruption ruins the doctor-patient relationship in India. BMJ 2014;348:g3169.

2. Mallath MK, Taylor DG, Badwe RA, Rath GK, Shanta V, Pramesh CS, et al. The growing burden of cancer in India: Epidemiology and social context. Lancet Oncol 2014;15:e205-12.

3. Pramesh CS, Badwe RA, Borthakur BB, Chandra M, Raj EH, Kannan $\mathrm{T}$, et al. Delivery of affordable and equitable cancer care in India. Lancet Oncol 2014;15:e223-33.

4. Collingridge D. Three countries-half of the global cancer burden. Lancet Oncol 2014;15:483.

5. Goss PE, Strasser-Weippl K, Lee-Bychkovsky BL, Fan L, Li J, Chavarri-Guerra $Y$, et al. Challenges to effective cancer control in China, India, and Russia. Lancet Oncol 2014;15:489-538.

6. Sankaranarayanan $R$, Swaminathan R, Brenner $H$, Chen K, Chia KS, Chen JG, et al. Cancer survival in Africa, Asia, and Central America: A population-based study. Lancet Oncol 2010;11:165-73.

7. Census of India: Census Data 2001: India at a glance $>>$ Rural-Urban Distribution. Office of the Registrar General and Census Commissioner, India. Available from: http:// censusindia.gov.in/Census_Data_2001/India_at_glance/. [Last retrieved on 2008 Nov 26].

8. Rath GK, Parikh PM, Hukku S, Rajan B, Kumar S, Malhotra H, et al. Indian Council of Medical Research consensus document for the management of buccal mucosa cancer. Indian J Med Paediatr Oncol 2014;35:136-9.

9. Pramesh CS, Badwe RA, Sinha RK. The national cancer grid of India. Indian J Med Pediatr Oncol 2014;35:226-7.

10. Available from: http://www.rcplondon.ac.uk/what-we-do/ patient-involvement/patient-and-carer-network. [Last accessed on 2014 Sep 5].

11. Bhawna G. Burden of smoked and smokeless tobacco consumption in India-Results from the Global adult Tobacco Survey India (GATS-India)-2009-201. Asian Pac J Cancer Prev 2013;14:3323-9.
12. Shastri SS, Mittra I, Mishra GA, Gupta S, Dikshit R, Singh S, et al. Effect of VIA screening by primary health workers: Randomized controlled study in Mumbai, India. J Natl Cancer Inst 2014;106:dju009.

13. Krishnan S, Madsen E, Porterfield D, Varghese B. Advancing cervical cancer prevention in India: Implementation science priorities. Oncologist 2013;18 Suppl:13-25.

14. National Cancer Registry Programme, Indian Council of Medical Research: Three year report of population based cancer registries 2009-2011. Available from: http://www. pbcrindia.org/.[Last accessed on 2014 June 16].

15. Mehrotra R, Gupta DK. Exciting new advances in oral cancer diagnosis: Avenues to early detection. Head Neck Oncol 2011;3:33.

16. Dreze J, Sen A. An uncertain glory-India and its contradictions. India: Penguin books Ltd.; 2013.

17. Mazor KM, Reed GW, Yood RA, Fischer MA, Baril J, Gurwitz JH. Disclosure of medical errors: What factors influence how patients respond? J Gen Intern Med 2006;21:704-10.

18. Scally G, Donaldson LJ. The NHS's 50 anniversary. Clinical governance and the drive for quality improvement in the new NHS in England. BMJ 1998;317:61-5.

19. Gabel M, Hilton NE, Nathanson SD. Multidisciplinary breast cancer clinics. Do they work? Cancer 1997;79:2380-4.

20. O'Dowd A. Doctors support biggest local reconfiguration in NHS, says Hunt. BMJ 2013;347:f6593.

21. Shrikhande SV, Barreto SG, Somashekar BA, Suradkar K, Shetty GS, TaloleS, etal. Evolution of pancreatoduodenectomy in a tertiary cancer center in India: Improved results from service reconfiguration. Pancreatology 2013;13:63-71.

22. Power LA, Anderson RW, Cortopassi R, Gera JR, Lewis RM Jr. Update on safe handling of hazardous drugs: The advice of experts. Am J Hosp Pharm 1990;47:1050-60.

23. Moore J, McQuestion M. The clinical nurse specialist in chronic diseases. Clin Nurse Spec 2012;26:149-63.

24. Parikh RB, Kirch RA, Smith TJ, Temel JS. Early specialty palliative care - translating data in oncology into practice. N Engl J Med 2013;369:2347-51.

25. Lubitz JD, Riley GF. Trends in medicare payments in the last year of life. N Engl J Med 1993;328:1092-6.

26. Joensuu $H$, Bono $P$, Kataja $V$, Alanko $T$, Kokko R, Asola R, et al. Fluorouracil, epirubicin, and cyclophosphamide with either docetaxel or vinorelbine, with or without trastuzumab, as adjuvant treatments of breast cancer: Final results of the FinHer Trial. J Clin Oncol 2009;27:5685-92.

27. Schnipper LE, Lyman GH, Blayney DW, Hoverman JR, Raghavan D, Wollins DS, et al. American Society of Clinical Oncology 2013 top five list in oncology. J Clin Oncol 2013;31:4362-70.

28. Schnipper LE, Smith TJ, Raghavan D, Blayney DW, Ganz PA, Mulvey TM, et al. American Society of Clinical Oncology identifies five key opportunities to improve care and reduce costs: The top five list for oncology. J Clin Oncol 2012;30:1715-24.

29. Sirohi B, Gupta S, Raghunadharao D, Shrikhande SV. Clinical trials in India: At uncertain crossroads? Indian J Med Paediatr Oncol 2014;35:133-5.

How to cite this article: Sirohi B. Cancer care delivery in India at the grassroot level: Improve outcomes. Indian J Med Paediatr Oncol 2014;35:187-91.

Source of Support: Nil, Conflict of Interest: None declared. 